\title{
Clinical evaluation of synthetic aperture harmonic imaging for scanning focal malignant liver lesions
}

Brandt, Andreas Hjelm; Hemmsen, Martin Christian; Hansen, Peter Møller; Madsen, Signe Sloth; Krohn, Paul Suno; Lange, Theis; Hansen, Kristoffer Lindskov; Jensen, Jørgen Arendt; Bachmann, Michael

Published in:

Ultrasound in Medicine \& Biology

Link to article, DOI:

10.1016/j.ultrasmedbio.2015.05.008

Publication date:

2015

Document Version

Peer reviewed version

Link back to DTU Orbit

Citation $(A P A)$ :

Brandt, A. H., Hemmsen, M. C., Hansen, P. M., Madsen, S. S., Krohn, P. S., Lange, T., Hansen, K. L., Jensen, J. A., \& Bachmann, M. (2015). Clinical evaluation of synthetic aperture harmonic imaging for scanning focal malignant liver lesions. Ultrasound in Medicine \& Biology, 41(9), 2368-75.

https://doi.org/10.1016/j.ultrasmedbio.2015.05.008

\section{General rights}

Copyright and moral rights for the publications made accessible in the public portal are retained by the authors and/or other copyright owners and it is a condition of accessing publications that users recognise and abide by the legal requirements associated with these rights.

- Users may download and print one copy of any publication from the public portal for the purpose of private study or research.

- You may not further distribute the material or use it for any profit-making activity or commercial gain

- You may freely distribute the URL identifying the publication in the public portal 


\title{
CLINICAL EVALUATION OF SYNTHETIC APERTURE HARMONIC IMAGING FOR SCANNING FOCAL MALIGNANT LIVER LESIONS
}

\author{
Andreas Huelm Brandt, ${ }^{*}$ Martin Christian Hemmsen, ${ }^{\dagger}$ Peter Møller Hansen, ${ }^{*}$ \\ Signe Sloth Madsen, ${ }^{*}$ Paul Suno Krohn, ${ }^{\ddagger}$ Theis Lange, ${ }^{\S}$ Kristoffer Lindskov Hansen, ${ }^{*}$ \\ Jørgen Arendt Jensen, ${ }^{\dagger}$ and Michael Bachmann Nielsen* \\ * Department of Radiology, Rigshospitalet, Copenhagen University Hospital, Copenhagen, Denmark; ${ }^{\dagger}$ Center for Fast \\ Ultrasound Imaging, Department of Electrical Engineering, Technical University of Denmark, Lyngby, Denmark; ${ }^{\ddagger}$ Department \\ of Surgical Gastroenterology, Rigshospitalet, Copenhagen University Hospital, Copenhagen, Denmark; and ${ }^{\S}$ Department of \\ Biostatistics, University of Copenhagen, Copenhagen, Denmark
}

(Received 25 February 2015; revised 13 April 2015; in final form 11 May 2015)

\begin{abstract}
The purpose of the study was to perform a clinical comparison of synthetic aperture sequential beamforming tissue harmonic imaging (SASB-THI) sequences with a conventional imaging technique, dynamic receive focusing with THI (DRF-THI). Both techniques used pulse inversion and were recorded interleaved using a commercial ultrasound system (UltraView 800, BK Medical, Herlev, Denmark). Thirty-one patients with malignant focal liver lesions (confirmed by biopsy or computed tomography/magnetic resonance) were scanned. Detection of malignant focal liver lesions and preference of image quality were evaluated blinded off-line by eight radiologists. In total, 2,032 evaluations of 127 image sequences were completed. The sensitivity (77\% SASB-THI, 76\% DRF-THI, $p=0.54)$ and specificity (71\% SASB-THI, 72\% DRF-THI, $p=0.67)$ of detection of liver lesions and the evaluation of image quality $(p=0.63)$ did not differ between SASB-THI and DRF-THI. This study indicates the ability of SASB-THI in a true clinical setting. (E-mail: andreaskr5@gmail.com) (c) 2015 World Federation for Ultrasound in Medicine \& Biology.
\end{abstract}

Key Words: Synthetic aperture, Sequential beamforming, Tissue harmonic imaging, Image evaluation, Liver lesion.

\section{INTRODUCTION}

Ultrasound plays a major role in medical imaging and is used for diagnosis and assessment in a variety of medical specialties. Hence, the improvement of ultrasound techniques will benefit a large group of patients and health care workers. Tissue harmonic imaging (THI) is an ultrasound technique that improves image resolution and contrast and provides gray-scale imaging with fewer artifacts (Averkiou et al. 1997; Tranquart et al. 1999; Ward et al. 1997). Combining conventional ultrasound algorithms with THI is therefore a standard method to improve the image quality of gray-scale imaging (Desser and Jeffrey 2001; Hann et al. 1999; Shapiro et al. 1998; Tranquart et al. 1999). However, conventional B-mode imaging techniques have several technical constraints, as images are acquired

Address correspondence to: Andreas Hjelm Brandt, Blegdamsvej 9, 2100 Copenhagen OE, Denmark. E-mail: andreaskr5@gmail.com sequentially one image line at a time. The frame rate is limited by the speed of sound in tissue, the scanning depth and the number of image lines. The high image resolution with a large number of image lines is, thus, obtained at the expense of frame rate. Image generation is further affected by a fixed transmit focus, causing the image to be optimally focused at only one depth. This can be improved by using multiple transmit foci, but the weakness of this solution is an increased number of emissions, which reduces the frame rate even further (Holm and Yao 1997).

High image resolution and high frame rate can be obtained with synthetic aperture (SA) (Sherwin et al. 1962). SA was originally developed from radar systems for geologic and sonar applications, but has been modified for medical imaging (Burckhardt et al. 1974). The basic idea underlying SA is generation of a highresolution image from a number of low-resolution images (Jensen et al. 2006). An active element is selected stepwise through the array. At each step, an unfocused 
beam is emitted, and all the elements in the array receive echoes to create the low-resolution images.

Several different implementations of SA exist. However, a common disadvantage hindering real-time implementation on a commercial scanner is the high system requirements (Behar and Adam 2005; Gammelmark and Jensen 2003; Karaman et al. 1995). Synthetic aperture sequential beamforming (SASB) was introduced to reduce the system requirements of SA. SASB is a dual-stage procedure using two separate beamformers (Kortbek et al. 2008). The first beamformer reduces the data throughput requirement to that of a single output signal, that is, a factor of 64 for a 64channel receive system. The second beamformer recombines a set of emissions to create the final high-resolution image (Hemmsen et al. 2012a; Kortbek et al. 2013). Previous studies have evaluated the image quality of SASB against conventional dynamic receive focusing (DRF) and reported equally good image quality, indicating that SASB is applicable to medical imaging (Hemmsen et al. 2011, 2012b, Hansen et al. 2014). SASB can generate an acoustic field intense enough to create harmonics for THI, and it has been suggested that these techniques be combined to improve the image quality of SASB even further. The pulse inversion technique was used to generate THI, and the beamforming steps for the final SASB-THI image are illustrated in Figure 1 (Hemmsen et al. 2014b; Rasmussen et al. 2012; Yigang et al. 2011). In a preliminary study in which healthy volunteers were scanned, two radiologists evaluated the image quality of SASB-THI as equal to that of a conventional imaging technique combined with THI (DRF-THI), indicating that SASB-THI can be used for medical imaging (Rasmussen et al. 2013).

The purpose of this study was to perform a clinical comparison of DRF-THI and SASB-THI using liver scans of patients with confirmed malignant focal liver cancer. The image sequences generated, SASB-THI and DRF-THI videos, were evaluated by radiologists for detection of malignant focal liver lesions and to assess the image quality of SASB-THI compared with that of DRF-THI in a clinical setting.

\section{METHODS}

\section{Patients}

Forty-three patients with different kinds of malignant focal liver cancer (primary liver tumor or liver metastasis) were asked to participate in the study. All patients were included after providing informed consent and on approval by the Danish National Committee on Biomedical Research Ethics (Journal No. H-1-2011124). Before the study, liver lesions were diagnosed by bi- opsy or computed tomography/magnetic resonance (CT/ MR). Surgery was scheduled the day after the ultrasound examination for all patients. Before the experimental scan, an orientation scan was performed with a conventional ultrasound scanner (UltraView 800, BK Medical, Herlev, Denmark), and if available, CT/MR was used to ensure correct scan position. Included were only patients in whom the pathology was visible on the orientation scan, which was performed without contrast enhancement. Twelve patients were excluded because the pathology was not visible; thus, a total of 31 patients with focal liver cancer ( 28 colorectal liver metastases and 3 hepatocellular carcinomas) were examined with the experimental setup. Among the patients examined were 10 women and 21 men, ranging in age from 37 to $82 \mathrm{y}$ (mean \pm standard deviation [SD]: $65.1 \pm 10.4 \mathrm{y}$ ) and in body mass index from 16.8 to $33.0 \mathrm{~kg} / \mathrm{m}^{2}$ (mean \pm SD: $24.7 \pm 4.4 \mathrm{~kg} / \mathrm{m}^{2}$ ).

\section{Scanning}

The patients were scanned in three positions where the liver lesions were visible and in three areas where no pathology was visible. The patients were positioned supine and were told to hold their breath and lie still during recording. All scans were performed by P.M.H. and A.H.B. The aim was to record six sequences for each patient, but because of technical challenges, this was possible for only 28 patients. One patient had only three recordings, and two patients had seven recordings because of errors made while saving and noticed after the scan session. A total of 185 image sequences were recorded.

The acoustic output of SASB-THI was determined before scanning. Intensities must be those recommended by the Food and Drug Administration (FDA) for abdominal scanning. The limits are given by the mechanical index, $\mathrm{MI} \leq 1.9$; the derated spatial peak, pulse average intensity, $I_{\text {sppa }} \leq 190 \mathrm{~W} / \mathrm{cm}^{2}$; and the derated spatial peak, temporal average intensity, $I_{\text {spta }} \leq 94 \mathrm{~mW} / \mathrm{cm}^{2}$ (Food and Drug Administration 2008). As SASB-THI and DRF-THI use the same transmit profile equal acoustic outputs are obtained. The intensities were $\mathrm{MI}=0.9$, $I_{\text {sppa }}=81.2 \mathrm{~W} / \mathrm{cm}^{2}$ and $I_{\text {spta }}=16.2 \mathrm{~mW} / \mathrm{cm}^{2}$ and, hence, were lower than the FDA limit.

\section{Equipment and data acquisition}

Experimental scans were performed with a conventional ultrasound scanner (UltraView 800, BK Medical, Herlev, Denmark) equipped with a research interface and an abdominal 3.5-MHz CL192-3 ML convex array transducer (Sound Technology, State College, PA, USA). The ultrasound scanner was connected to a stand-alone PC. With the experimental setup, images generated with SASB-THI and DRF-THI were recorded 


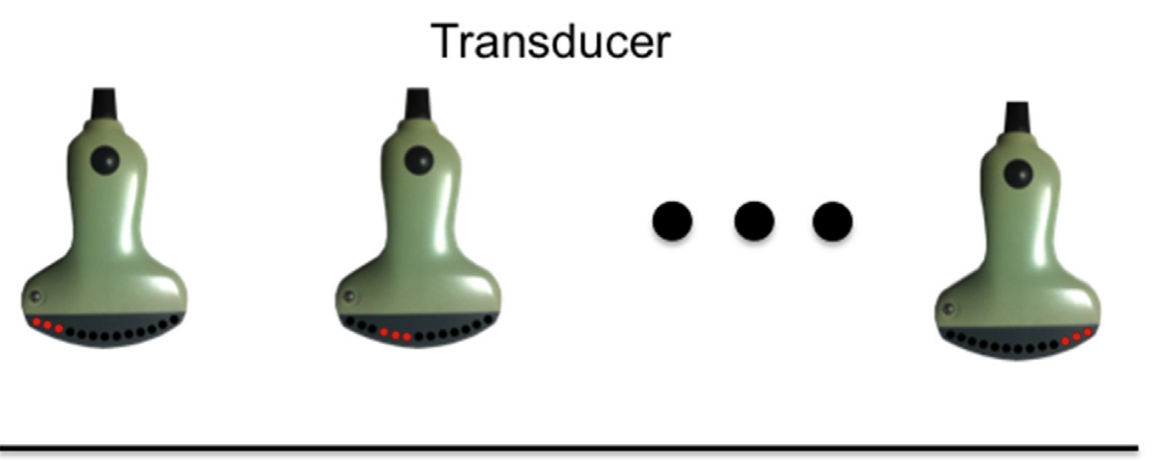

\section{Low resolution SASB-THI images}
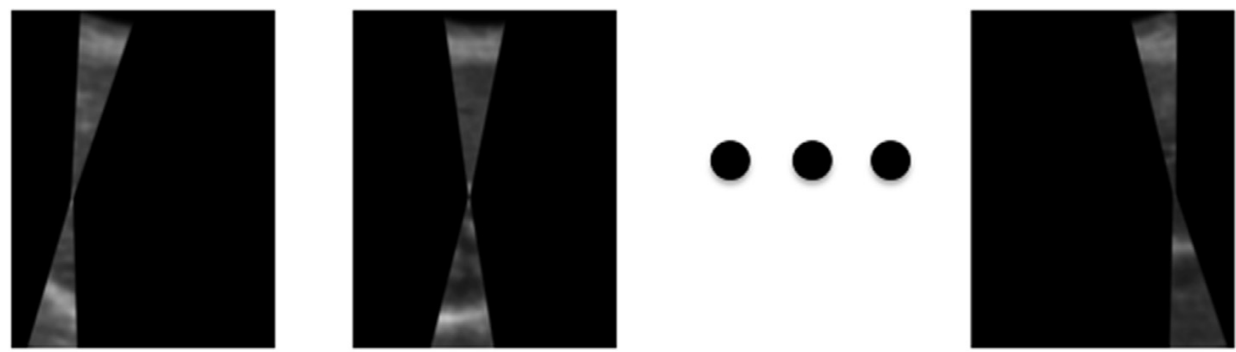

\section{Summation}

\section{High resolution SASB-THI image}

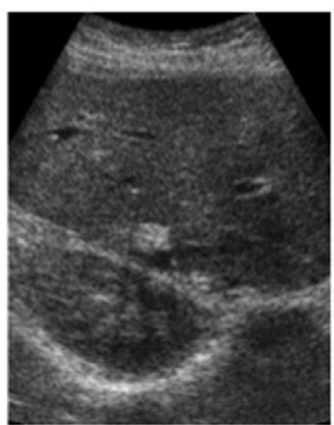

Fig. 1. Beamforming steps in achieving synthetic aperture sequential beamforming tissue harmonic imaging (SASBTHI). Transmit and receive elements are identical for each emission. Even harmonics (THI) are enhanced by the pulse inversion technique, and each beam is perceived as a virtual ultrasound source emitting from the beam focal point.

The received beams are summed in the second-stage beamformer to yield the high-resolution SASB-THI image.

interleaved. One frame generated with SASB-THI followed one frame generated with DRF-THI. Images from the same anatomic location were thereby recorded almost simultaneously with both techniques, and ideal sequences for comparison were generated (Hemmsen et al. 2010, 2012c).
The first beamforming of the dual-stage beamforming of SASB-THI was performed on the conventional scanner, and data were then recorded on the PC. The second beamforming was performed on the PC using MATLAB (The MathWorks, Natick, MA, USA) and the in-house developed beamformation toolbox BFT3 
(Hansen et al. 2011). DRF-THI images were entirely generated by the conventional scanner. Field-of-view, time-gain compensation, frame rate ( 8 frames/s), apodization and depth $(14.6 \mathrm{~cm})$ were identical for the two techniques. Three-second image sequences were generated. During the recording, only images for navigational purposes from the first beamforming were visualized on the scanner.

The navigational image had a low frame rate and poor image quality and was therefore merely used for guidance during collection of data. The final image sequences were available off-line after second-stage beamforming. To ensure that clinically valuable image sequences were acquired, a subsequent selection was performed before the image evaluation. Images defined as not clinically valuable were (i) sequences in which no liver tissue was visible, (ii) sequences in which malignant focal liver cancer was not visible even though it had been reported and (iii) sequences in which patient movement made the sequence impossible to assess. The selection was done blinded to knowledge of image technique. Nevertheless, both SASB-THI and DRF-THI sequences were affected, as both techniques were processed from the same data. A total of 58 image sequences with the same number of images for each technique were removed. This corresponds to $31.4 \%$ of all recorded sequences; therefore, 127 image sequences remained for image evaluation (Fig. 2). Patients in the excluded sequences were similar in age (45-78 y, mean \pm SD: $66.5 \pm 7.8 \mathrm{y})$ and body mass index $\left(18.6-33.0 \mathrm{~kg} / \mathrm{m}^{2}\right.$, mean $\left.\pm \mathrm{SD}: 24.6 \pm 4.4 \mathrm{~kg} / \mathrm{m}^{2}\right)$ to the patients whose sequences were included.

\section{Image evaluation}

Eight radiologists (examiners 1-8) blinded to the technical information evaluated all image sequences. The radiologists were asked to evaluate whether images contained malignant focal liver lesions, so that detection rates (sensitivity and specificity) could be determined. They were informed that some of the images contained malignant focal liver lesions. An in-house developed software program (IQap) was used for the evaluation (Hemmsen et al. 2010). Images obtained with the two techniques were shown separately, resulting in 254 evaluations by each radiologist for a total of 2,032 evaluations

The same eight radiologists also compared the image quality of both imaging techniques. This was similarly performed with the IQap. For each image sequence, the images were displayed side-by-side. The evaluating radiologist had the option of viewing the sequences in real time and as single frames. Each sequence was shown twice and randomly switched from left to right, displaying each technique twice. By placing a sliding bar on a visual analogue scale (VAS) (Freyd 1923) (Fig. 3), radiologists indicated the sequence with their preferred image quality as previously described by Hansen et al. (2014). The VAS ranged from -50 to 50, and positive values always favored SASB-THI, regardless of the side on which the SASB-THI image was placed. The values on the VAS scale were not shown on the scale during the evaluation and were therefore arbitrary for the evaluator. By sliding the bar further to one side or the other, the evaluator indicated his or her preference for that technique. By placing the bar in the middle, the evaluator indicated no difference between the techniques. Each evaluated image sequence was given an integer or numbered zero if the evaluators found no difference.

\section{Statistics}

Detection of focal malignant liver lesions was assessed by calculating sensitivity and specificity. Confidence intervals for sensitivity and specificity, as well as $p$ values for differences, were computed by bootstrapping to respect the complex dependence structures in the data. Inter-observer variability was calculated using Fleiss' $\kappa$ statistic, and $\kappa$ values were interpreted as proposed by Landis and Koch (1977) for strength of agreement: $\leq 0=$ poor, $0.01-0.20=$ slight, $0.21-0.40=$ fair, $0.41-0.60=$ moderate, $0.61-0.80=$ substantial and $0.81-1=$ almost perfect.

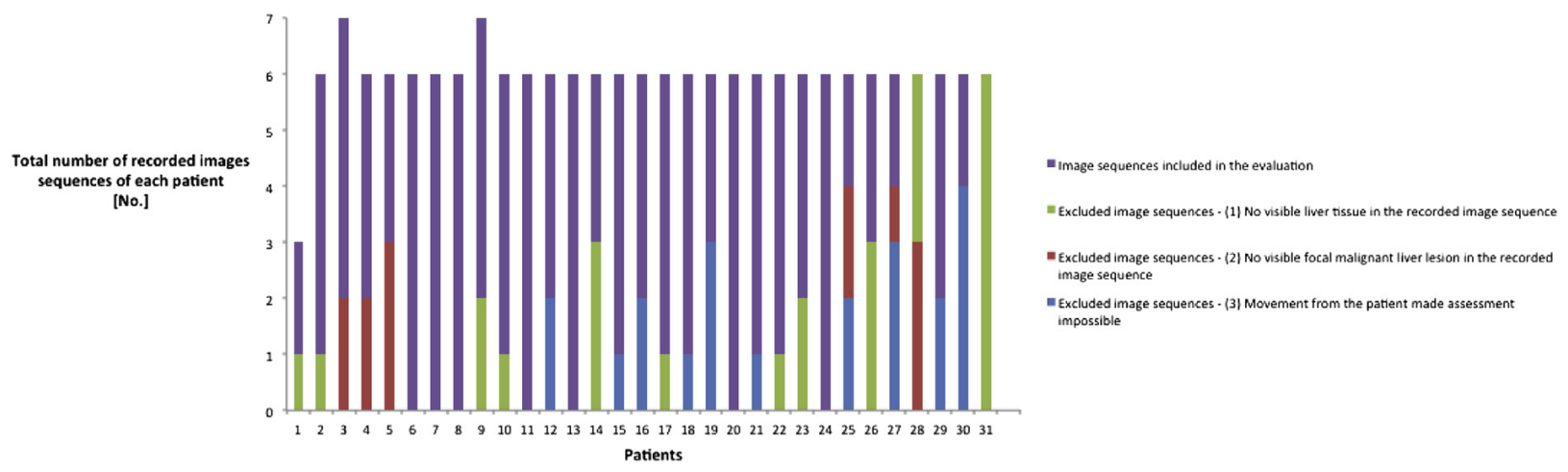

Fig. 2. Overview of image sequences included and excluded in the image evaluation. 


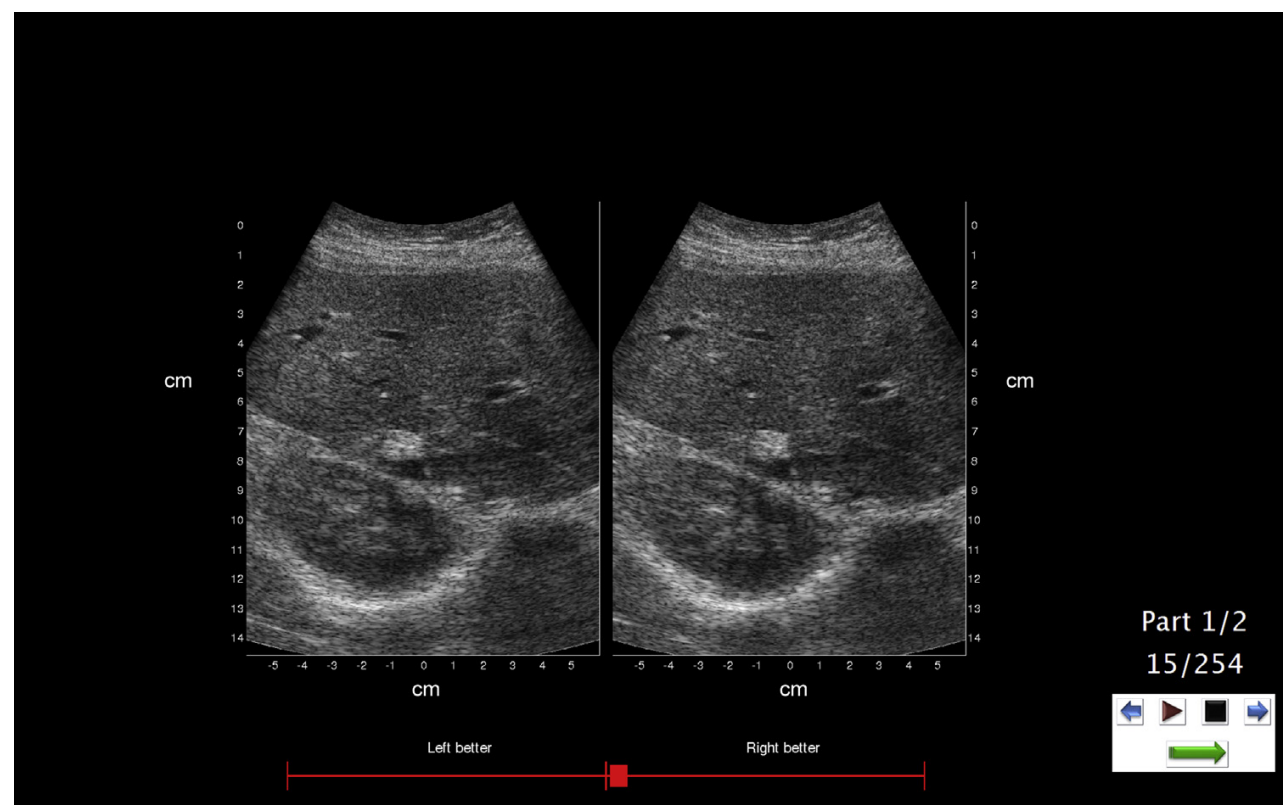

Fig. 3. IQap screenshot with the visual analogue scale at the bottom. A focal liver lesion is seen just above the kidney. By dragging the bar to one side, evaluators specified which technique they preferred. Left: sequential beamforming tissue harmonic imaging (SASB-THI), right: dynamic receive focusing with tissue harmonic imaging (DRF-THI).

In the evaluation of image quality, a non-parametric Wilcoxon signed rank test with bootstrapping was used to test the hypothesis of no difference in preference. This test takes into account that the same pair of images is displayed twice to the same radiologists and that the same image pairs are shown to different radiologists. Furthermore, the test handles the difficulties resulting from each radiologist having his or her own interpretation of the VAS scale. A linear mixed model was applied to test the same hypothesis, in the subgroups of radiologists who seemed to use the VAS similarly. As all image pairs were shown with SASB-THI images both on the left side and on the right side, it was not necessary to control for left/right differences. For the evaluation of image quality, inter-observer and intra-observer variability was determined using Fleiss' $\kappa$ statistic.

Data management was performed using Excel (Microsoft, Redmond, WA, USA) and MATLAB. Statistical analyses were performed using the statistical data analysis language $\mathrm{R}$, Version 2.12.2 (http://www. r-project.org/).

\section{RESULTS}

A focal malignant liver lesion was present in 55 image sequences, whereas 72 image sequences revealed only healthy liver tissue. The sensitivity and specificity of detection of focal malignant liver lesions are illustrated in Figure 4. Both imaging techniques had similar sensitivity and specificity; that is, there were no significant differences in mean sensitivity and specificity be- tween SASB-THI (sensitivity: 77\%, 95\% confidence interval [CI]: 70\%-84\%; specificity: $71 \%$, 95\% CI: 66\%-77\%) and DRF-THI (sensitivity: 76\%, 95\% CI: 69\%-82\%; specificity $72 \%$, 95\% CI: $67 \%-77 \%)$ ( $p=$ 0.54 [sensitivity] and 0.67 [specificity]). Inter-observer variability between the radiologists indicated moderate agreement $(k=0.48)$ when rating image sequences generated by SASB-THI and fair agreement $(k=0.37)$ when rating images generated with DRF-THI.

The image quality preference evaluation of each radiologist is illustrated in Figure 5. There was no preference for SASB-THI or DRF-THI in 63\% $(1,271 / 2032)$ of the evaluations, SASB-THI was favored by $16 \%$ (329/ 2032) and DRF-THI was favored by $21 \%$ (432/2032). The average rating for all radiologists was $-0.10(95 \%$ CI: -0.47 to 0.26 ), indicating no difference in preference for an imaging technique $(p=0.63)$. Inter-observer variability indicated poor agreement $(k=0.0045)$, and intraobserver variability indicated slight agreement $(k=0.11)$.

Radiologist 8 (Fig. 5) used the VAS scale more broadly then radiologists 1-7. An additional analysis, excluding radiologist 8 , yielded an average rating of $0.045(95 \% \mathrm{Cl}:-0.15$ to 0.24$)$, again indicating no preference for one technique $(p=0.62)$.

\section{DISCUSSION}

To our knowledge, this is the first study to examine use of the combination of SASB and THI on patients. Thirty-one patients with focal malignant liver lesions diagnosed by biopsy or MR/CT and scheduled for surgery 

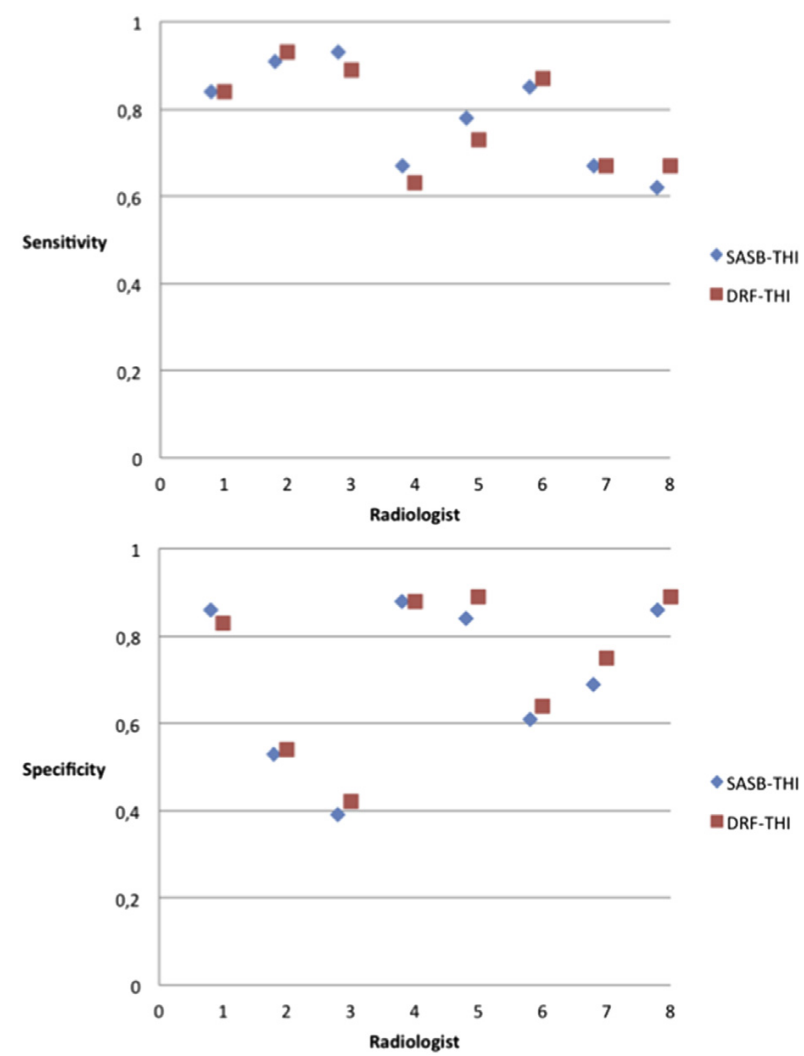

Fig. 4. Sensitivity and specificity of sequential beamforming tissue harmonic imaging (SASB-THI) and dynamic receive focusing with tissue harmonic imaging (DRF-THI) for each radiologist.

the day after the experimental examination were included in the study. Eight radiologists evaluated SASB-THI and DRF-THI sequences of livers with and without focal malignant liver lesions to assess diagnostic sensitivity and specificity, as well as image quality. The findings from this study, together with previous theoretical and experimental reports (Hemmsen et al. 2014a; Rasmussen et al. 2013), suggest that SASB-THI can be used for medical imaging.

With respect to sensitivity and specificity, the techniques performed equally well (Fig. 4), indicating that SASB-THI has the same detection rate as a conventional imaging technique when evaluating images for malignant focal liver lesions. Reasonable sensitivity and specificity values were obtained with both techniques, as the rate of detection of metastases with unenhanced ultrasound has been reported to have a sensitivity of $50 \%-76 \%$ and specificity of 60\%-96\% (Beissert et al. 2000; Cantisani et al. 2014; Glover et al. 2002) and detection of hepatocellular carcinoma has been reported to have a sensitivity as low as $33 \%-57 \%$ and a specificity of $80 \%-92 \%$ (Kim et al. 2001; Shapiro et al. 1996). Sensitivity and specificity can be improved by the use of ultrasound contrast (Cantisani et al. 2014), which will be pursued in future studies.
With respect to image quality, $63 \%$ of the evaluations were rated alike, and statistical analysis indicated that the radiologists did not have a preference for one technique $(p=0.63)$. One radiologist used the VAS differently than the other examiners. To compensate for this, the analysis was conducted both with and without this radiologist, with no change in the results. The similar image quality of SASB-THI and DRF-THI may explain the low inter-observer and intra-observer variability, as radiologists were unable to distinguish images obtained with SASB-THI and DRF-THI, and their choices were thus solely coincidences.

Until now, few clinical studies have been performed with SA as the imaging technique. Previous studies have evaluated the quality of still images of patients with focal breast pathology (Kim et al. 2012, 2013). The major advantage of the present study is the possibility of reviewing real-time sequences and single frames. In our opinion, this is a more reliable evaluation, because ultrasound is a dynamic examination. Furthermore, in this setup, the sequences were recorded interleaved and the same anatomic areas were compared, as opposed to previous studies in which the different images were recorded one after the other (Sodhi et al. 2005; Yen et al. 2008).

In a previous pre-clinical study with SASB-THI as the imaging technique, only healthy slim volunteers were scanned (Rasmussen et al. 2013). Scanning heavy or obese patients is more difficult, because of the thicker abdominal fat layers and higher heart rates (Hansen et al. 2014). Several patients in this study were hard to scan as they had discomfort lying on their backs, an altered anatomic layout because of previous surgery, trouble holding their breath and trouble lying still. Combined with the coarse navigation image, which was displayed while data were recorded, these problems made scanning difficult and were the main reasons for excluding $31.4 \%$ of the sequences from the final experimental data. In our opinion, however, this does not diminish the results of this study, as both SASB-THI and DRF-THI images were similarly affected, and the decisions to exclude images were made without knowledge of the imaging technique used.

A disadvantage of synthetic aperture imaging systems is tissue motion artifacts, although these artifacts have been found to have a minor impact on image quality (Jensen et al. 2006; Pedersen et al. 2007). Requesting patients to hold their breath and lie still most likely reduced these artifacts. Some tissue motion was evident in the image sequences evaluated and no degradation of image quality was seen, which is consistent with previous findings with clinical SASB imaging (Hansen et al. 2014). However, this study did not evaluate tissue motion with beamformed SASB-THI images, which should be evaluated in future clinical studies. 
Examiner 1

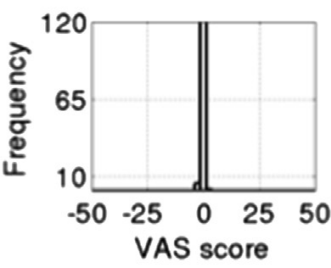

Examiner 5

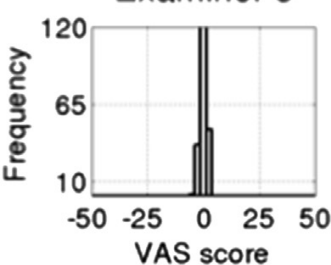

Examiner 2

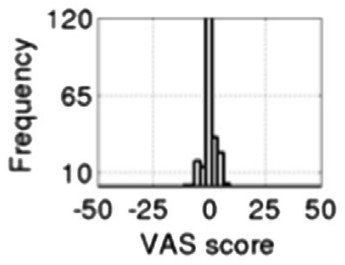

Examiner 6

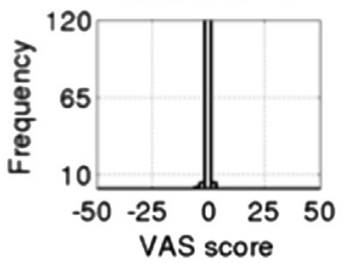

Examiner 3

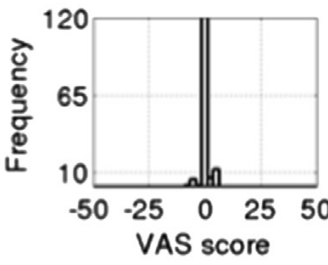

Examiner 7

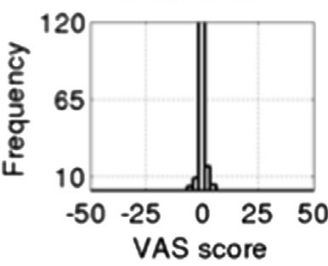

Examiner 4

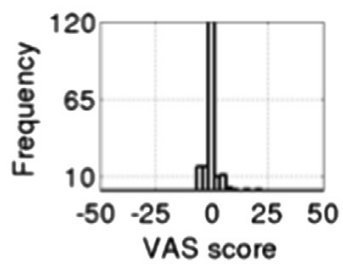

Examiner 8

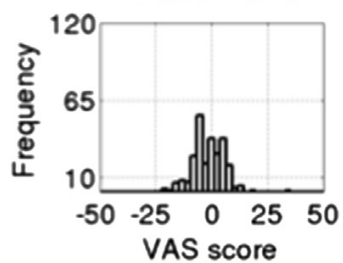

Fig. 5. Distribution of each radiologist's evaluations. Sequential beamforming tissue harmonic imaging is favored by positive values. VAS $=$ visual analogue scale.

All images were recorded at a relatively low frame rate without any image-improving algorithms, for example, speckle reduction filter, or image compounding. The images containing malignant focal liver lesions were therefore not optimized for diagnostic evaluation. Radiologists were told not to appraise the correct diagnosis, but only to detect the presence of malignant focal liver lesions. Future studies including different imageimproving algorithms and using ultrasound contrast with SASB-THI will reveal more about the diagnostic accuracy of SASB-THI.

Apart from the high frame rate and high resolution achieved, the predominant advantage of SASB-THI is a factor of 64 times lower data transmission between the probe and processing unit compared with conventional imaging. This reduction in data transmission does not lower the quality of the image and supports the use of SASB-THI for clinical imaging. The reduction in data transmission indicates the possibility of implementing a synthetic aperture technique on a commercially available hand-held tablet and producing wireless transducers (Hemmsen et al. 2014a). A wireless transducer can improve clinical conditions, as it makes it easier to scan directly at the trauma site, makes it easier to maintain sterile conditions, simplifies ultrasound-guided intervention and peri-operative scanning, improves freedom of movement and optimizes awkward and ergonomically challenging positions (Munoz and Zamorano 2014). Combining the wireless transducer with a commercial tablet would spread the use of ultrasound tremendously, as tablets are relatively inexpensive and widely available. Moreover, easily maneuverable hand-held devices, like tablets, have proven to be useful in numerous clinical conditions (Lapostolle et al. 2006). In cardiology, in particular, hand-held devices have facilitated rapid diagnosis and patient screening in good agreement with conventional ultrasound systems (Biais et al. 2012).

\section{CONCLUSIONS}

Synthetic aperture sequential beamforming tissue harmonic imaging has successfully been used in a clinical setting. Patients with malignant focal liver cancer were scanned, and interleaved image sequences were recorded with both SASB-THI and DRF-THI. In a double-blinded setup, eight ultrasound-experienced radiologists rated SASB-THI equal to DRF-THI with respect to ability to detect malignant focal liver lesions and image quality. This indicates that SASB-THI can be used in the clinical setting. The advantage of the reduction in data transmission can be used to implement wireless real-time SASBTHI on a commercial available hand-held tablet.

Acknowledgments - The authors thank all participating patients at the Department of Surgical Gastroenterology, Rigshospitalet, and the staff for helping to recruit patients. Also, special thanks to Professor Lars Bo Svendsen for letting us scan in the department. Thanks to Flemming Jensen, M.D., Caroline Ewertsen, M.D., Trine-Lise Lambine, M.D., Mikkel Seidelin Dam, M.D., Dorte Stærke, M.D., Masoud Azapour, M.D., and Lars Lönn, M.D., for evaluating the ultrasound recordings. The study was supported by Grant 82-2012-4 from the Danish National Advanced Technology Foundation and by BK Medical ApS.

\section{REFERENCES}

Averkiou MA, Roundhill DN, Powers JE. A new imaging technique based on the nonlinear properties of tissues. Proc IEEE Ultrason Symp 1997;2:1561-1566.

Behar V, Adam D. Optimization of sparse synthetic transmit aperture imaging with coded excitation and frequency division. Ultrasonics 2005;43:777-788.

Beissert M, Jenett M, Keberle M. Comparison of contrast harmonic imaging in B-mode with stimulated acoustic emission, conventional B- 
mode US and spiral CT in the detection of focal liver lesions. Rofo 2000; $172: 361-366$

Biais M, Carrie C, Delaunay F, Morel N, Revel P, Janvier G. Evaluation of a new pocket echoscopic device for focused cardiac ultrasonography in an emergency setting. Crit Care 2012;16:R82.

Burckhardt CB, Grandchamp PA, Hoffmann H. An experimental $2 \mathrm{MHz}$ synthetic aperture sonar system intended for medical use. IEEE Trans Son Ultrason 1974;21:1-6.

Cantisani V, Grazhdani H, Fioravanti C, Rosignuolo M, Calliada F, Messineo D, Bernieri MG, Redler A, Catalano C, D'Ambrosio F. Liver metastases: Contrast-enhanced ultrasound compared with computed tomography and magnetic resonance. World J Gastroenterol 2014;20:9998-10007.

Desser TS, Jeffrey RB. Tissue harmonic imaging techniques: Physical principles and clinical applications. Semin Ultrasound CT MR 2001;22:1-10.

Food and Drug Administration (FDA). Guidance for industry and FDA staff-Information for manufacturers seeking marketing clearance of diagnostic ultrasound systems and transducers. Technical Report. U.S. Department of Health and Human Services, FDA, Center for Devices and Radiologic Health, http://www.fda.gov/Medical Devices/. 2008.

Freyd M. The graphic rating scale. J Educ Psychol 1923;14:83-102.

Gammelmark KL, Jensen JA. Multielement synthetic transmit aperture imaging using temporal encoding. IEEE Trans Med Imaging 2003; 22:552-563.

Glover C, Douse P, Kane P, Karani J, Meire H, Mohammadtaghi S, Allen-Mersh T. Accuracy of investigations for asymptomatic colorectal liver metastases. Dis Colon Rectum 2002;45:476-484.

Hann LE, Bach AM, Cramer LD, Siegel D, Yoo HH, Garcia R. Hepatic sonography: Comparison of tissue harmonic and standard sonography techniques. AJR Am J Roentgenol 1999;173:201-206.

Hansen JM, Hemmsen MC, Jensen JA. An object-oriented multithreaded software beamformation toolbox. Proc SPIE 2011;7968.

Hansen PM, Hemmsen M, Brandt A, Rasmussen J, Lange T, Krohn PS, Lonn L, Jensen JA, Nielsen MB. Clinical evaluation of synthetic aperture sequential beamforming ultrasound in patients with liver tumors. Ultrasound Med Biol 2014;40:2805-2810.

Hemmsen MC, Petersen MM, Nikolov SI, Nielsen MB, Jensen JA. Ultrasound image quality assessment: A framework for evaluation of clinical image quality. Proc SPIE 2010;7629.

Hemmsen MC, Hansen PM, Lange T, Hansen JM, Nikolov SI, Nielsen MB, Jensen JA. Preliminary in-vivo evaluation of synthetic aperture sequential Beamformation using a multielement convex array. Proc IEEE Ultrason Symp 2011;1131-1134.

Hemmsen MC, Hansen JM, Jensen JA. Synthetic aperture sequential beamformation applied to medical imaging. Proc EUSAR 2012a;34-37.

Hemmsen MC, Hansen PM, Lange T, Hansen JM, Hansen KL, Nielsen MB, Jensen JA. In vivo evaluation of synthetic aperture sequential beamforming. Ultrasound Med Biol 2012b;38:708-716.

Hemmsen MC, Kjeldsen T, Larsen L, Kjær C, Tomov BG, Mosegaard J, Jensen JA. Implementation of synthetic aperture imaging on a handheld device. Proc IEEE Ultrason Symp 2014a;2177-2180.

Hemmsen MC, Nikolov SI, Pedersen MM, Pihl MJ, Enevoldsen MS, Hansen JM, Jensen JA. Implementation of a versatile research data acquisition system using a commercially available medical ultrasound scanner. IEEE Trans Ultrason Ferroelectr Freq Control 2012c;59:1487-1499.

Hemmsen MC, Rasmussen J, Jensen JA. Tissue harmonic synthetic aperture ultrasound imaging. J Acoust Soc Am 2014b;136:2050-2056.

Holm S, Yao H. Improved framerate with synthetic transmit aperture imaging using prefocused subapertures. Proc IEEE Ultrason Symp 1997;2:1535-1538.

Jensen JA, Nikolov SI, Gammelmark KL, Pedersen MH. Synthetic aperture ultrasound imaging. Ultrasonics 2006;44(Suppl 1):e5-e15.
Karaman M, Pai-Chi L, O'Donnell M. Synthetic aperture imaging for small scale systems. IEEE Trans Ultrason Ferroelectr Freq Control 1995:42:429-442.

Kim C, Yoon C, Park JH, Lee Y, Kim WH, Chang JM, Choi BI, Song TK, Yoo YM. Evaluation of ultrasound synthetic aperture imaging using bidirectional pixel-based focusing: Preliminary phantom and in vivo breast study. IEEE Trans Biomed Eng 2013;60: 2716-2724.

Kim CK, Lim JH, Lee WJ. Detection of hepatocellular carcinomas and dysplastic nodules in cirrhotic liver: Accuracy of ultrasonography in transplant patients. J Ultrasound Med 2001; 20:99-104.

Kim WH, Chang JM, Kim C, Park J, Yoo Y, Moon WK, Cho N, Choi BI. Synthetic aperture imaging in breast ultrasound: A preliminary clinical study. Acad Radiol 2012;19:923-929.

Kortbek J, Hensen JA, Gammelmark KL. Synthetic aperture sequential beamforming. Proc IEEE Int Ultrason Symp 2008;966-969.

Kortbek J, Jensen JA, Gammelmark KL. Sequential beamforming for synthetic aperture imaging. Ultrasonics 2013;53:1-16.

Landis JR, Koch GG. The measurement of observer agreement for categorical data. Biometrics 1977;33:159-174.

Lapostolle F, Petrovic T, Lenoir G, Catineau J, Galinski M, Metzger J, Chanzy E, Adnet F. Usefulness of hand-held ultrasound devices in out-of-hospital diagnosis performed by emergency physicians. Am J Emerg Med 2006;24:237-242.

Munoz DR, Zamorano JL. Wireless echocardiography: A step towards the future. Eur Heart J 2014;35:1700.

Pedersen MH, Gammelmark KL, Jensen JA. In-vivo evaluation of convex array synthetic aperture imaging. Ultrasound Med Biol 2007;33:37-47.

Rasmussen J, Hemmsen MC, Madsen SS, Hansen PM, Nielsen MB, Jensen JA. Implementation of tissue harmonic synthetic aperture imaging on a commercial ultrasound system. Proc IEEE Ultrason Symp 2012;121-125.

Rasmussen J, Hemmsen MC, Madsen SS, Hansen PM, Nielsen MB, Jensen JA. Preliminary study of synthetic aperture tissue harmonic imaging on in vivo data. Proc SPIE 2013;8675.

Shapiro RS, Katz R, Mendelson DS, Halton KP, Schwartz ME, Miller CM. Detection of hepatocellular carcinoma in cirrhotic patients: Sensitivity of CT and ultrasonography. J Ultrasound Med 1996;15:497-502.

Shapiro RS, Wagreich J, Parsons RB, Stancato-Pasik A, Yeh HC, Lao R. Tissue harmonic imaging sonography: Evaluation of image quality compared with conventional sonography. AJR Am J Roentgenol 1998;171:1203-1206.

Sherwin CW, Ruina JP, Rawcliffe RD. Some early developments in synthetic aperture radar systems. IRE Trans Mil Elect 1962; MIL-6:111-115.

Sodhi KS, Sidhu R, Gulati M, Saxena A, Suri S, Chawla Y. Role of tissue harmonic imaging in focal hepatic lesions: Comparison with conventional sonography. J Gastroenterol Hepatol 2005;20: $1488-1493$.

Tranquart F, Grenier N, Eder V, Pourcelot L. Clinical use of ultrasound tissue harmonic imaging. Ultrasound Med Biol 1999;25: 889-894.

Ward B, Baker AC, Humphrey VF. Nonlinear propagation applied to the improvement of resolution in diagnostic medical ultrasound. J Acoust Soc Am 1997;101:143-154.

Yen CL, Jeng CM, Yang SS. The benefits of comparing conventional sonography, real-time spatial compound sonography, tissue harmonic sonography, and tissue harmonic compound sonography of hepatic lesions. Clin Imaging 2008;32:11-15.

Yigang D, Rasmussen J, Jensen H, Jensen JA. Second harmonic imaging using synthetic aperture sequential beamforming. Proc IEEE Ultrason Symp 2011;2261-2264. 